\title{
26301 - LEARNING CURVES FOR ULTRASOUND-GUIDED REGIONAL ANESTHESIA
}

\section{Lawrence Lou MD, Ban Tsui, MD; University Of Alberta, Edmonton, ALBERTA, Canada}

INTRODUCTION: The use of ultrasound (U/S) imaging for regional anesthesia is an emerging and important field in anesthesiology. It can improve both the rate and quality of regional blocks (1). Substantial time and effort must be devoted to learn how to efficiently use U/S to obtain a suitable anatomic image. We hypothesize that certain approaches for using U/S during regional blocks have a more prolonged learning curve than others and may not be suitable for inexperienced clinicians. The purpose of this study was to examine anesthesiology and radiology residents' ability to learn and use U/S to identify the brachial plexus from commonly used interscalene and supraclavicular approaches.

METHODS: Following IRB approval, 16 anesthesiology (PGY1-4) and 14 radiology (PGY2-4) residents, with no prior ultrasound -guided regional anesthesia experience, were given a brief presentation on the use of U/S during brachial plexus blocks using both an interscalene and a supraclavicular approach. Residents were randomly assigned sealed envelopes containing instructions to find the brachial plexus with one of these approaches. Each resident was allowed up to 5 minutes to read the instructions and a maximum of 5 minutes to obtain their clearest U/S picture of the brachial plexus on a volunteer. Success rate and time to find the correct image was recorded. Each resident was then given instructions for the alternate approach and the procedure was repeated. The same volunteer was used for each session. The same qualified anesthesiologist judged U/S image accuracy.

RESULTS: As shown in Table 1, the anesthesiology residents had much higher success, in a shorter length of time, with the supraclavicular approach. While this is the only statistically significant difference, the interscalene approach was notably easier for the radiology residents.

DISCUSSION: The time and success rate for the anesthesiology residents to identify the brachial plexus using U/S was significantly shorter and higher (respectively) using a supraclavicular approach over the interscalene approach. However, such a difference was not observed for the radiology residents. Despite this, radiology residents used the interscalene approach faster and more successfully than the anesthesiology residents, although this was not statistically significant and was likely due to the small sample size. These results suggest that viewing a readily identifiable vascular structure (i.e. the subclavian artery) may be more helpful for the naive anesthesiology resident than the more imaging inclined radiology residents. In summary, a supraclavicular approach with U/S may be more suitable for inexperienced clinicians when identifying the brachial plexus image as it appears to be more reliable than the interscalene approach. In contrast, those with U/S experience can likely use either approach effectively.

REFERENCE:

1) Anesth Analg 2003;97: 1518-23 
Table 1 Success and Rate of Using Ultrasound for Viewing the Brachial Plexus

\begin{tabular}{|c|c|c|c|c|}
\hline & \multicolumn{2}{|c|}{ Interscalene Approach } & \multicolumn{2}{c|}{ Supraclavicular Approach } \\
\hline Discipline & Time (SD) & $\%$ Success & Time (SD) & \% Success \\
\hline Anesthesiology & $206.6(121.5)$ & 50 & $53.6(75.0)^{*}$ & $100^{*}$ \\
\hline Radiology & $128.1(103.6)$ & 78.6 & $82.4(83.2)$ & 93.8 \\
\hline
\end{tabular}

* Supraclavicular versts Interscalene approach for anesthesiologist residents: significance $\mathrm{P}<0.05$ 